\title{
Postnatal paternal depressive symptoms associated with fathers' subsequent parenting: findings from the Millennium Cohort Study
}

\author{
Selina Nath, Ginny Russell, Tamsin Ford, Willem Kuyken and Lamprini Psychogiou
}

\section{Summary}

Impaired parenting may lie on the causal pathway between paternal depression and children's outcomes We use the first four surveys of the Millennium Cohort Study to investigate the association between paternal depressive symptoms and fathers' parenting (negative, positive and involvement). Findings suggest that postnatal paternal depressive symptoms are associated with fathers' negative parenting. This has implications for the design of intervention programmes for parents with depression and young children.

\section{Declaration of interest}

None.

\section{Copyright and usage}

(C) The Royal College of Psychiatrists 2015.
Impaired parenting may be an influence along the causal pathway between paternal depression and children's developmental outcomes. ${ }^{1}$ Therefore, it is important to understand which aspects of parenting behaviours are influenced by paternal depressive symptoms. A meta-analysis of 28 studies reported paternal depression to be associated with reduced positive and increased negative parenting behaviours. ${ }^{2-3}$ Using the Millennium Cohort Study (MCS), Malmberg \& Flouri ${ }^{4}$ reported that higher levels of paternal depressive symptoms when the children were 9 months old were associated with lower overall positive father-child relationship quality at 3 years old. Furthermore, an Australian study suggested that higher levels of paternal depressive symptoms might influence fathers' negative parenting rather than positive parenting. ${ }^{5}$ Higher postnatal paternal depressive symptoms were associated with increased hostility towards children at 5 years old, but no association with warmth was detected. The current study aimed to test whether higher levels of paternal depressive symptoms were independently associated with positive parenting (warmth), negative parenting (conflict) or the amount of time devoted to parenting activities (involvement), after controlling for a number of possible covariates, and to test whether any of these covariates may moderate any such association.

\section{Method}

Secondary data analysis was carried out using the first four waves of the MCS. This is a large-scale $(n=18552)$ survey of infants when they were 9 months (MCS1), 3 years (MCS2), 5 years (MCS3) and 7 years old (MCS4). Full details of the measures, survey, objectives, content of survey and sampling can be found elsewhere. ${ }^{6-8}$

The predictor variable was paternal depressive symptoms at MCS1 measured using the Rutter's 9-item Malaise Inventory (shortened version). ${ }^{9,10}$ Higher scores on the scale between 0 and 9 indicated more depressive symptoms. The outcome measure of fathers' parenting at MCS2 was assessed using the Child-Parent Relationship Scale, ${ }^{11}$ which measured two constructs of fatherchild relationship based on the father's report of warmth and conflict. Fathers' parenting activity (involvement) was measured as outcomes at MSC3 and MSC4 using fathers' answers to the amount of parenting activities they undertook with their child. Items were summed to create a total score of fathers' parenting activity at both sweeps with higher scores indicating less involvement and lower scores indicating more involvement.
Covariates were family and socioeconomic factors including continuous scales of maternal depressive symptoms, child temperament, marital relationship, family income, paternal age and dichotomous scales for child gender (boy/girl), paternal education (with qualifications/no education), fathers' employment (employed/unemployed), family housing (tenants/property owners). All measures were reported at MCS1 ( 9 months) and have all been found to have an influence on fathers' depression., ${ }^{4,12,13}$

Sampling weights were used in analyses that adjust for complex sample design and attrition over time to make it representative of the UK population as a whole. ${ }^{8,14}$ Analyses were conducted using Stata 13. Linear regressions were conducted to test the relationship of paternal depressive symptoms (predictor) with father-child conflict/warmth relationship (outcomes at MCS2) and fathers' parenting activities (outcome at MCS3 and MCS4). Covariates were selected $a$ priori based on previous literature. ${ }^{4,5,12,13}$ To check whether family context and socioeconomic covariates were associated with the outcomes, a further series of linear regressions were conducted. Covariates that were significantly $(P<0.05)$ associated with fathers' parenting outcomes were taken forward into a series of adjusted multivariable linear regression models to test whether the predictor paternal depressive symptoms (MCS1) were independently associated with father-child warmth/conflict relationship and fathers' parenting activities. Each outcome was tested in a separate model. Sensitivity analysis was conducted using multiple imputation to determine the effect of missing data. The following covariates were tested for moderating effects based on previous literature: ${ }^{1,4,5,12}$ maternal depressive symptoms, child temperament, child gender, marital relationship, fathers' employment and age.

\section{Results}

In the unadjusted analysis, higher paternal depressive symptoms significantly predicted higher levels of father-child conflict, lower warmth scores and lower levels of involvement (online Table DS1). Descriptive statistics for the outcome variables are provided in online Table DS2. All covariates apart from fathers' employment status were significantly associated with fathers' parenting activities and all apart from education were also significantly associated with father-child conflict and warmth. After adjustment for potential confounders and weighted to account for survey design and attrition, higher levels of paternal depressive 
symptoms were independently associated with more father-child conflict. This finding was also replicated with un-weighted analysis and imputed data (online Table DS3). The overall model explained $16 \%$ of the variance in father-child conflict $\left(R^{2}=0.16\right)$.

Only maternal depressive symptoms (coefficient $-0.11,95 \%$ CI -0.18 to $-0.03, P=0.006$ ) and marital conflict (coefficient $0.12,95 \%$ CI -0.24 to $-0.01, P=0.037$ ) appeared to moderate the association between paternal depressive symptoms and father-child conflict after adjustment. The influence of paternal depressive symptoms on father-child conflict scores was lower in families with high marital conflict and when mothers had high depressive symptoms, than for families with low marital conflict and mothers with fewer depressive symptoms (online Figs DS1 and DS2).

\section{Discussion}

The findings show that paternal depressive symptoms may be associated with higher levels of father-child conflict, which replicates previous findings 5,15 and suggests that paternal depressive symptoms influence negative, rather than positive parenting. Paternal depressive symptoms were not associated with paternal involvement, suggesting that the quality of parenting is influenced by depressive symptoms but the duration of time spent with the child is not altered. Both maternal depressive symptoms and marital conflict moderated the association between paternal depressive symptoms and father-child conflict, whereas withinchild factors did not. Paternal depressive symptoms were associated with more father-child conflict in families with low marital conflict and where mothers had lower levels of depressive symptoms. Maternal influence on fathers' parenting style replicate previous literature ${ }^{4,12}$ and the current findings suggest that in families with low marital conflict and low levels of depressive symptoms in mothers, fathers might be more available to have negative interactions with their children. Thus, this might increase the likelihood of more father-child conflict potentially resulting from their depressive symptoms. This finding is not expected from previous maternal and paternal literature, ${ }^{1}$ but it fits well with our findings that paternal depressive symptoms were not associated with a reduction of father's parenting activities. Thus, fathers' depressive symptoms may not reduce their involvement, but influences the quality of their interactions with their children. However, when there are high levels of maternal depressive symptoms combined with high levels of marital conflict, fatherchild conflict may be lower, because negative interaction might become focused on the mother rather than the child. Moreover, previous research suggests that paternal depressive symptoms influence maternal depressive symptoms, which may increase children's emotional and behavioural problems. ${ }^{1,4}$ Thus, paternal depressive symptoms might also indirectly influence children via effects on their mothers. Although this is an interesting finding, these results may have been because of the use of conventional regression analysis of effect modification, which assumes linear relationships between variables based on mean responses in the sample population. Thus, further research is needed.

Our study has a number of strengths. First, the MCS collected data on a very large representative sample of fathers in the UK. Second, the study used well-validated measures and maintained a high response rate. ${ }^{7,8}$ Finally, sampling weights were applied to account for stratified sampling and attrition. ${ }^{8,14}$ Findings however, were based solely on self-report questionnaires from fathers. Results could have been affected by shared method bias; that is, fathers with depressive symptoms may be more likely to report negative than positive behaviours. Additionally, no clinical diagnoses for depression were available. However, findings from our secondary data analysis studies can inform future experimental and longitudinal studies in clinical samples that could include direct observation and structured diagnostic assessment. If our findings were replicated, parenting interventions for fathers with depression should focus on the reduction of father-child conflict as well as promoting positive parenting. Practitioners should consider the needs of the partners of parents they treat for depression in terms of support they might need.

Despite reports showing the huge costs of paternal depression, parenting interventions are still primarily targeted towards mothers. ${ }^{16}$ Taking our findings into account, we advocate a more family-centred approach and provided that appropriate support and services are put in place, we would suggest routine screening for postnatal depressive symptoms in fathers, as is currently the case for mothers. Additionally, further studies need to be undertaken to establish whether fathers' elevated scores on screening measures constitute depression or a normal but difficult adjustment phase. In both cases, support for fathers need to be put in place.

Selina Nath, PhD, Institute of Psychiatry, Psychology and Neuroscience, King's
College London, London; Ginny Russell, PhD, Tamsin Ford, MRCPsych, PhD,
University of Exeter Medical School, Willem Kuyken, PhD, DClinPsy, Department
of Psychiatry, Warneford Hospital, Oxford; Lamprini Psychogiou, PhD, University
of Exeter, Exeter, UK

Correspondence: Selina Nath, Section of Women's Mental Health, PO31 Institute of Psychiatry, Psychology and Neuroscience, King's College London, De Crespigny Park, London SE5 8AF, UK. Email: selina.nath@kcl.ac.uk

First received 14 Mar 2014, final revision 12 Mar 2015, accepted 13 Mar 2015

\section{References}

1 Ramchandani P, Psychogiou L. Paternal psychiatric disorders and children's psychosocial development. Lancet 2009; 374: 646-53.

2 Wilson S, Durbin CE. Effects of paternal depression on fathers' parenting behaviors: a meta-analytic review. Clin Psychol Rev 2010; 30: 167-80.

3 Davis RN, Davis MM, Freed GL, Clark SJ. Fathers' depression related to positive and negative parenting behaviors with 1-year-old children. Pediatrics 2011; 127: 612-8.

4 Malmberg LE, Flouri E. The comparison and interdependence of maternal and paternal influences on young children's behavior and resilience. J Clin Child Adolesc Psychol 2011; 40: 434-4.

5 Giallo R, Cooklin A, Wade C, D'Esposito, Nicholson JM. Fathers' postnatal mental health and child wellbeing at age 5 : the mediating role of parenting behaviour. J Fam Issues 2014; 35: 1543-62.

6 Johnson J. Millennium Cohort Study: Psychological, Developmental and Health Inventories. Centre for Longitudinal studies, Institute of Education, 2012.

7 Hansen K. Millennium Cohort Study First, Second, Third and Fourth Surveys: A Guide to the Datasets (7th edn). Centre for Longitudinal Studies, Institute of Education, 2012.

8 Plewis I, Ketende S. Millennium Cohort Study: Technical Report on Responses (1st edn). Centre for Longitudinal Studies, Institute of Education, 2006.

9 Dex S, Joshi H. Millennium Cohort Study First Survey: A User's Guide to Initial Findings. Centre for Longitudinal studies, Institute of Education, 2004.

10 Rutter M, Tizard J, Whitmore K. Education, Health And Behaviour. Longman Publishing Group, 1970.

11 Pianta RC. Child-Parent Relationship Scale. University of Virginia, 1995

12 Giallo R, D'Esposito F, Cooklin A, Mensah F, Lucas N, Wade C, et al. Psychosocial risk factors associated with fathers' mental health in the postnatal period: results from a population-based study. Soc Psychiatry Psychiatr Epidemiol 2013; 48: 563-73.

13 Bruder-Costello B, Warner V, Talati A, Normura Y, Bruder G, Weissman M. Temperament among offspring at high and low risk for depression. Psychiatry Res 2007; 153: 145-51.

14 Ketende SC, Jones EM. Millennium Cohort Study: User Guild to Analysing MCS Data using Stata. Centre for Longitudinal studies, Institute of Education, 2011.

15 Kane $\mathrm{P}$, Garber J. The relations among depression in fathers, children's psychopathology, and father-child conflict: a meta-analysis. Clin Psychol Rev 2004; 24: 339-60.

16 Edoka IP, Petrou S, Ramchandani P. Healthcare costs of paternal depression in the postnatal period. J Affect Disord 2011; 133: 356-60. 